\title{
Aktivitäten steigern Zufriedenheit und Wohlbefinden von Heimbewohnern
}

\begin{abstract}
Wie schätzen Pflegeheimbewohner ihr emotionales Erleben, ihr körperliches und psychosoziales Wohlbefinden ein, wie sehr fühlen sie sich durch psychosomatische Störungen und Beschwerden subjektiv beeinträchtigt und was bedeutet dies für ihre Lebensqualität? Eine Befragung im Rahmen des Projekts „Bündnis 70 plus“ untersucht psychosoziale Faktoren aus Sicht der Bewohner.
\end{abstract}

$\mathrm{D}$ ie Studie der Nürnberger Universitätsklinik der Paracelsus Medizinischen Privatuniversität untersucht den Zusammenhang von Befinden und psychosozialen Aktivitäten im Pflegeheim und vergleicht Aussagen der Bewohner mit Bewertungen von Pflegepersonal aus anderen wissenschaftlichen Studien. Ziel der Studie ist es, spezifische Empfehlungen für Pflegeheime abzuleiten.

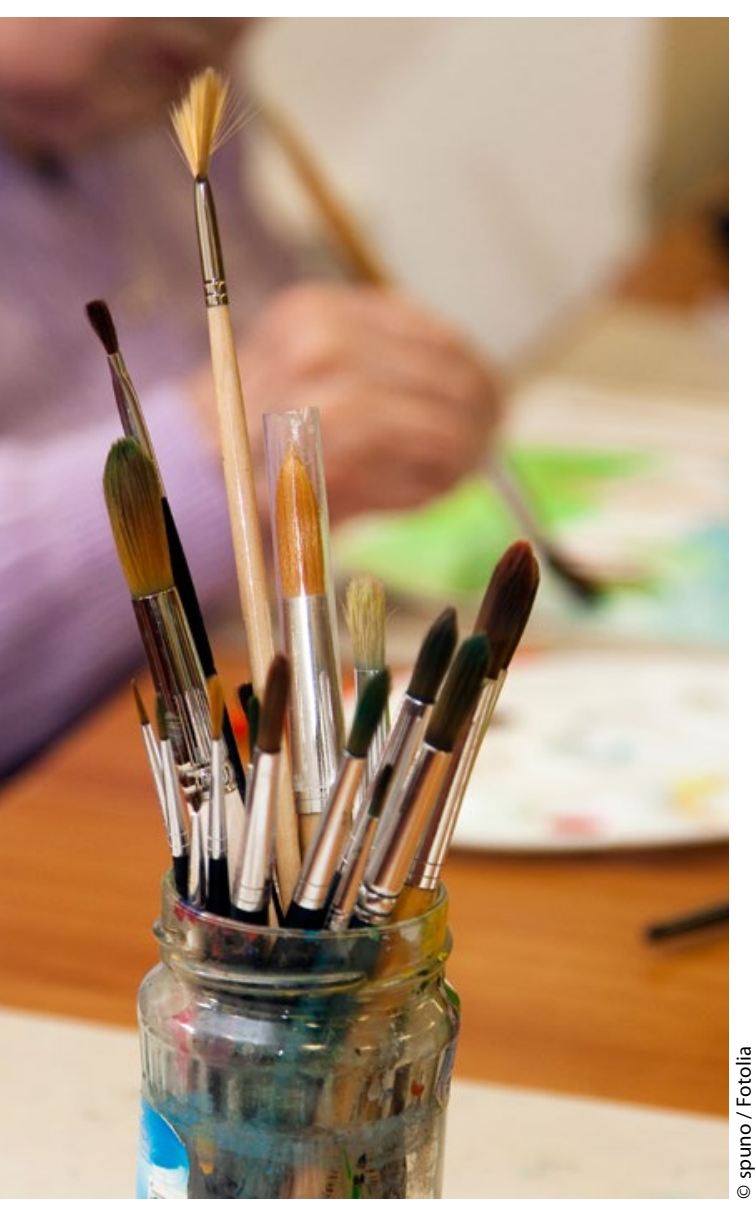

Die regelmäßige Teilnahme an Heimangeboten und anderen Aktivitäten fördert die Lebenszufriedenheit.
Die Querschnittsstudie wurde mittels semistrukturierter, anonymisierter Interviews durchgeführt. Parallel dazu wurden die Teilnehmer testpsychologisch hinsichtlich einer depressiven und demenziellen Symptomatik untersucht (Screeningverfahren: Kurzform der Geriatric Depression Scale / GDS-K bzw. Mini Mental Status Test / MMST).

Studienteilnehmer waren 256 Bewohner in zwei Nürnberger Pflegeheimen mit einem Durchschnittsalter von 81 Jahren (SD $\pm 10,3$ Jahre). 176 waren Frauen (69\%), 80 Männer (31\%). Sie wurden zu ihren körperlichen, psychischen und sozialen Aktivitäten befragt. Mehr als die Hälfte der Heimbewohner schätzte ihren Gesundheitszustand als gut bis sehr gut ein. Dabei gaben sie in der persönlichen Befragung ein nahezu doppelt so häufiges regelmäßiges Schmerzempfinden an als es laut einer Vergleichsstudie von Pflegekräften registriert wurde. Bei $44,6 \%$ der Bewohner gab es Hinweise auf eine depressive Symptomatik, bei $76,1 \%$ auf eine demenzielle. Eine signifikante Korrelation mit der subjektiven Einschätzung der Lebenszufriedenheit zeigte sich unter den Studienteilnehmern bei den Items Teilnahme an Heimaktivitäten ( $r=0,171 ; p=0,008)$, Mobilität $(r=-0,131 ; p=0,045)$, emotionale Aktivität $(\mathrm{r}=0,135 ; \mathrm{p}=0,038)$, körperlicher Gesundheitszustand $(r=-0,420$; $p$ $<0,001)$, regelmäßiges Schmerzempfin$\operatorname{den}(r=-0,178 ; p=0,006)$. Bedeutsam ist die Korrelation der Lebenszufriedenheit mit depressiven Symptomen $(r=-0,617$; $\mathrm{p}<0,001)$ und der kognitiven Leistungsfähigkeit $(r=0,251 ; p=0,001)$. Unter den Frauen war die subjektiv geäußerte Lebenszufriedenheit insgesamt höher als bei den Männern. Die Ergebnisse, so die Autoren, lassen vermuten, dass die regelmäßige Teilnahme an Heimangeboten und anderen Aktivitäten die Lebenszufriedenheit fördert und dass zufriedene Bewohner ihren Gesundheitszustand positiver einschätzen, sich mobiler fühlen und weniger Schmerzen empfinden.

Sabine M. Kempa

Cramer-Ebner R et al. Befinden und psychosoziale Aktivitäten im Pflegeheim Befragung von Bewohnern. Z Gerontol Geriat 2016 First Online: 28 June 2016; doi 10.1007/s00391-016-1080-7

\section{Kommentar}

Beschäftigungsangebote steigern die Zufriedenheit der Heimbewohner/innen, so eines der Ergebnisse der vorliegenden Studie. Das ist bei der doch sehr unterschiedlichen Qualität der Ergo-Angebote überaus erstaunlich. Von läppisch bis höchst ambitioniert reicht bei den Beschäftigungsangeboten in Heimen die NiveauPalette. Gestandene Heimbewohner erhalten da schon mal Ausmalbögen, so als ob sie kleine Kinder seien. Umso mehr die Bewohner/innen aufgrund ihrer Multimorbidität zur Passivität verdammt sind, desto mehr werden die „Ergos" zu Egos: In einer noblen Residenz erscheint da beim Themenabend "Venedig" der Beschäftigungsassistent im Rokoko-Livree. Aber es gibt auch einige sehr gute Angebote, beispielsweise die professionell begleitete "Generationenbrücke“", bei der ein Jahr lang Viertklässler eine Seniorengruppe im Heim besuchen, um mit ihnen zu singen, sich zu bewegen, zu plaudern und zu basteln. Dann fegt ein ganz schön frischer Wirbelwind durch das Heim. Die Angebote sind also unterschiedlich angemessen. Und daher ist es nicht verkehrt, wenn in weiteren Studien herausgearbeitet wird, wie „Persönlichkeitsstrukturen und Interessenschwerpunkte der Heimbewohner" bei den Beschäftigungsangeboten besser berücksichtigen werden können.

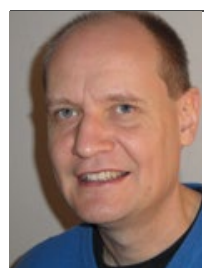

Markus Hieber Diplom-Pflegewirt, Altenpfleger Luitpoldstr. $15 \mathrm{~A}$ 0781 Berlin markus_hieber@t-online. de 